\title{
Association between suicidal ideation and causes of primary care visits: A cross-sectional study to identify patients with suspected suicidal ideation
}

\section{Gyeongsil Lee}

Seoul National University Hospital https://orcid.org/0000-0003-1910-9658

Jung-Ha Kim ( $\sim$ girlpower219@cau.ac.kr)

https://orcid.org/0000-0002-7630-9501

\section{Research article}

Keywords: Suicide ideation, Suicide prevention, Primary care, Mental disorder, Musculoskeletal disorder

Posted Date: June 17th, 2019

DOI: https://doi.org/10.21203/rs.2.10353/v1

License: (c) (1) This work is licensed under a Creative Commons Attribution 4.0 International License.

Read Full License

Version of Record: A version of this preprint was published at Psychiatry Investigation on July 15th, 2020. See the published version at https://doi.org/10.30773/pi.2020.0048. 


\section{Abstract}

Background: Evidence increasingly suggests that suicide victims are highly likely to visit a primary care provider within one month of the suicide event. However, it would be impossible for primary care providers to act as gatekeepers and thus screen all patients for suicidal ideation or attempts. Therefore, we aimed to investigate potential differences in the chief diagnoses received by primary care patients with and without suicidal ideation, as this information may provide clues and predict patients at risk of committing suicide. Methods: This cross-sectional study included 1,211 Korean adults aged $\geq 20$ years who had participated in the Korea National Health and Nutrition Examination Survey during 2014 and provided information about their histories of suicidal ideation and visits to primary care providers.

Multiple logistic regression analyses were used to examine whether suicidal ideation was associated with specific diseases after controlling for age, household income, education, occupation, marital status, smoking, alcohol consumption and physical activity. Results: Among primary care patients, the sampleweighted percentage of suicidal ideation was $11.8 \%$. The diagnoses of neoplasm and mental disorder were associated with a higher likelihood of suicidal ideation (odds ratio; OR [95\% confidence interval, Cl]: 8.32 [1.01-68.56] and 6.19 [1.72-22.33], respectively). Notably, diseases of the musculoskeletal system and connective tissue were also associated with suicidal ideation (OR [95\% Cl]: 1.93 [1.09-3.43]). Conclusions: The observed visit patterns and correlations of certain diagnoses with suicidal ideation suggest that primary care physician should pay careful attention to their patients with mood or musculoskeletal disorders and consider screening to determine the risk of suicide.

\section{Background}

Suicide is a critical global public health issue [1], particularly in the Republic of Korea where the prevalence of suicide is among the highest in the world. According to a report from the Korea Suicide Prevention Center, the national prevalence of suicide increased steadily from 16.6 to 33.5 per 100,000 people during 2000-2010, and decreased slightly thereafter to 25.8 per 100,000 people in 2015 [2]. In contrast, the average prevalence of suicide among Organization for Economic Cooperation and Development (OECD) countries in 2015 was 11.8 per 100,000 people [2]. This discrepancy highlights the status of suicide as a public health crisis in Korea.

The above-described suicide mortality patterns have elicited a range of preventive strategies worldwide. Several previous reports stressed comprehensive and integrated multidisciplinary approaches to the reduction of suicide and suicidal behavior [1, 3-5], among which gatekeeper training is regarded as one of the most effective strategies. Gatekeepers are individuals who come into frequent contact with members of the community, including primary, mental and emergency health care providers; teachers and other school staff; community leaders and police officers; and other first responders [1]. Some studies reported that approximately $45 \%$ of suicide victims had contact with a primary care provider within one month of the suicide event [6]. This evidence suggests that primary care providers could play a prominent role in suicide prevention [7]. However, it would be impossible for primary care physicians to screen all visiting patients for suicidal ideation or attempts, as the general population is not considered a high-risk group. 
The U.S. Preventive Services Task Force (USPSTF) also concluded that "the current evidence is insufficient to assess the balance of benefits and harms of screening for suicide risk in adolescents, adults, and older adults in primary care [8]." In summary, the prevention of suicide would rely on the identification of patients who are likely to commit suicide.

The Korean National Health and Nutrition Examination Survey (KNHANES) includes data about suicidal ideation, the primary care visit history, and information about diagnoses made during the most recent 2week period. These data could form the basis of an investigation of differences in the chief complaints and diagnoses of primary care patients with and without suicidal ideation. The results of such an investigation could subsequently facilitate the prediction of patients at risk of committing suicide. Therefore, this study aimed to determine the association between suicidal ideation and the primary care visit history, using data from the KNHANES in 2014.

\section{Methods}

\subsection{Study participants}

This cross-sectional study used data from the KNHANES, a nationwide survey of non-institutionalized civilian residents of South Korea that has been conducted by the Korean Ministry of Health and Welfare since 1998. Briefly, this nationwide representative study uses a stratified, multistage probability sampling design to select household units [9]. The KNHANES aims to evaluate the nutritional and health status of the general population and subsequently provide representative and reliable statistical public health data that can be used as a basis for health care policies.

This analysis was based on data obtained from 5,428 respondents to the 2014 survey who were aged $\geq$ 20 years. From this initial population, we excluded the following individuals for the following reasons: no information about outpatient visits during the most recent 2-week period $(n=3,670)$; no visits to a primary care center $(n=536)$; and missing data for the ninth question on the Patient Health Questionnaire (PHQ-9) $(n=11)$. The present study included 1,211 Korean adults.

\subsection{Study variables}

The PHQ-9 is a reliable and valid screening tool used widely to detect depressive disorders, including suicidal ideation, in the general population [10]. The Korean version of PHQ-9, which is also considered reliable and valid, was included in the KNHNES VI-2 survey [11]. In this study, we defined a participant as having suicidal ideation if they provided a positive response (i.e., "yes") to the ninth item on the PHQ-9, which asked "Have you ever been thought that you would be better off dead, or of hurting yourself in recent two weeks?"

Diseases that served as the causes for visits to primary care providers were identified via diagnostic information and had been recorded by physicians according to the Tenth International Classification of 
Diseases (ICD-10). The following codes and classifications were applied: A00-B99, Certain infectious and parasitic diseases; $\mathrm{C00}-\mathrm{C} 97$, Neoplasms; E00-E90, Endocrine, nutritional and metabolic diseases; F00-F99, Mental and behavioral disorders; G00-G99, Diseases of the nervous system; H0O-H59, Diseases of the eye and adnexa; H60-H95, Diseases of the ear and mastoid process; 100-199, Diseases of the circulatory system; J00-J99, Diseases of the respiratory system; K00-K93, Diseases of the digestive system; L00-L99, Diseases of the skin and subcutaneous tissue; M00-M99, Diseases of the musculoskeletal system and connective tissue; N00-N99, Diseases of the genitourinary system; O00099, Pregnancy, childbirth and the puerperium; R00-R99, Symptoms, signs and abnormal clinical and laboratory findings, not elsewhere classified; S00-T98, Injury, poisoning and certain other consequences of external causes; and Z00-Z99, Factors influencing health status and contact with health services. We also identified self-reported underlying diseases, including hypertension, diabetes mellitus, dyslipidemia, stroke, cardiovascular disease (angina or myocardial infarction), depression, and cancer diagnosed by a medical doctor.

The potential confounders included age, gender, residence, household income, education, marital status, body mass index (BMI), current smoking, alcohol consumption, and physical activity. Residence was classified as urban or rural. Household income was classified into quartiles. Education status was classified into two categories: high school or lower and college or higher. Occupations were classified as follows: manager, professional or office worker; service or sales; manual worker and unemployed (e.g., housewife or student). Cohabitation status was classified as either living alone or living together. BMI was derived from the measured weight and height and reported in units of $\mathrm{kg} / \mathrm{m}^{2}$. Obesity was defined as a BMI of $\geq 25 \mathrm{~kg} / \mathrm{m}^{2}$ according to the current Asian obesity guidelines [12]. A current smoker was defined as someone who had smoked $\geq 100$ cigarettes ( 5 packs) throughout his or her lifetime and continued to smoke at the time of the survey. A heavy drinker was defined as a person who consumed at least one bottle of soju (Korean distilled spirits) during a 1-week period. Regular physical activity was defined as participation in vigorous activity (e.g., running or mountaineering) for $\geq 75 \mathrm{~min} /$ week or moderate intensity activity (e.g., light swimming, badminton or walking) for $\geq 150 \mathrm{~min} /$ week [13]. Strength exercise was defined as the performance of exercise at least twice a week.

\subsection{Statistical analysis}

The survey procedures in SAS 9.2 (SAS Institute Inc., Cary, NC, USA) were applied to complex sampling designs. The characteristics of the study population are reported as means \pm standard errors (SEs) for continuous variables and as weighted percentages (\%) with SEs for categorical variables. We categorized participants into two groups according to suicidal ideation, using the ninth item of the PHQ-9. Differences in ICD-10 codes among the two groups were analyzed using a weighted t-test and chi-square test. Multiple logistic regression analyses were used to examine the potential associations of suicidal ideation with specific diseases after controlling for age, household income, education, occupation, marital status, smoking, alcohol habit and physical activity. Statistical significance was set at a two-sided $p$ value $<0.05$. 


\section{Results}

Table 1 presents the general characteristics of the study population, stratified by suicidal ideation. The sample-weighted frequency of suicidal ideation among primary care patients was $11.8 \%$. Patients who expressed suicidal ideation were more likely to be female, have low income and education levels, live alone, and be unemployed and less likely to perform strength exercise. Table 2 presents information about disease diagnoses made by primary care providers during the 2-week period before the survey according to ICD-10 codes, as well as self-reported underlying diseases, according to the suicidal ideation status. Of the ICD-10 coded diseases, mental and behavioral disorders (F00-F99) and diseases of the musculoskeletal system and connective tissue (M00-M99) were significantly more frequent among individuals with suicidal ideation. Among self-reported underlying diseases, hypertension, stroke, and depression were significantly more frequent among individuals with suicidal ideation.

Table 3 presents the significant multivariable adjusted odds ratios (ORs) with $95 \%$ confidence intervals (Cls) of ICD-10 coded diseases and suicidal ideation. Notably, diagnoses of neoplasm (C00-C97) and mental disorder (F00-F99) were associated with the greatest increases in the probability of suicidal ideation (OR [95\% Cl]: 8.32 [1.01-68.56] and 6.19 [1.72-22.33], respectively), although diseases of the musculoskeletal system and connective tissue (M00-M99) were also associated with suicidal ideation (OR [95\% Cl]: 1.93 [1.09-3.43]). Conversely, injury, poisoning, and certain other consequences of external causes (S00-T98) were associated with a lack of suicidal ideation (OR [95\% Cl]: 0.17[10.04-0.77]). We further calculated the frequency of visits to primary care providers during the most recent 2-week period. In an analysis stratified by ICD-10 codes assigned by primary care providers, injury, poisoning, and certain other consequences of external causes were significantly more frequent among individuals without suicidal ideation $(p<0.01)$. No significant associations were observed between other diseases diagnosed by primary care providers and the presence of suicidal ideation.

\section{Discussion}

In this cross-sectional study of data from the KNHANES, we identified that approximately $12 \%$ of primary care patients reported suicidal ideation. This rate was higher than the range of $1.0-10.5 \%$ reported by a review of suicidal ideation among primary care patients [14] and similar to the rates from German primary care studies in which the PHQ was used to assess suicidal ideation [15]. Moreover, the primary care patients with suicidal ideation in this study were more likely to be women, have lower income and education levels, live alone, and be unemployed, consistent with multiple reports describing risk factors for suicidal ideation $[16,17]$. We further investigated potential differences in the chief primary care diagnoses of patients with and without suicidal ideation and found that the former were more likely to receive diagnoses of neoplasms (C00-C97), mental and/or behavioral disorders (F00-F99), and diseases of the musculoskeletal system and connective tissue (M00-M99), while the latter were more likely to be diagnosed with injury, poisoning, and certain other consequences of external causes (S00T98). 
Previous studies reported that primary care patients with suicidal ideation were more frequently diagnosed with depression [18] and anxiety disorders [19], compared to those without suicidal ideation. Consistent with those findings, we observed a higher frequency of diagnosed mental and behavioral disorders (F00-F99) among patients with suicidal ideation. This finding is highly plausible, as a previous systematic review of psychological autopsy studies of suicide reported that $>60 \%$ of suicidal deaths involved victims who had experienced major depressive disorder or other mood disorders [20]. Moreover, patients with neoplasms (C00-C97) have a high risk of psychological problems [21], consistent with our findings.

Additionally, we identified diseases of the musculoskeletal system and connective tissue (M00-M99) as primary causes of visits to primary care providers among individuals with suicidal ideation. Although no previous studies evaluated the association between musculoskeletal disorder and suicidal ideation, a review of psychological risk factors associated with back and neck pain reported that psychological factors play an important role not only in chronic pain, but also in the etiology of acute pain, particularly during the transition to chronic problems [22]. Accordingly, patients with physical pain due to musculoskeletal disorders may experience depressive symptoms, which would explain the observed significant association with suicidal ideation.

Our analysis revealed that injury, poisoning, and certain other consequences of external causes were diagnosed more frequently in patients without suicidal ideation. In contrast, a previous study based on Korean national health insurance database investigated medical facility visit histories according to suicidal death [23], and found that in addition to those with mental and behavioral disorders (F00-F99), patients who visited a medical facility for injury, poisoning, and certain other consequences of external causes (S00-T98) had a significantly increased risk of suicide death. We attribute this inter-study difference to variations in medical facilities and disease severity levels. In the earlier study, disease information was solicited from all types of medical facilities, including psychiatric departments and emergency departments. In this study, however, we investigated only disease information from primary care patients with suicidal ideation, rather than suicidal death. Possibly, injured patients relating to suicidal attempt more frequently visited the emergency departments of general hospitals due to its severity. Despite these differences in study participants and disease severity, all medical facilities reported a higher probability of suicidal ideation among patients diagnosed with mental and behavioral disorders (F00-F99). In other words, all types of mental disorders should be addressed cautiously, regardless of severity.

The identified visit patterns suggest that primary care physicians should act as gatekeepers and suggest that patients with mental or musculoskeletal disorders consider undergoing screening for suicidal ideation. Currently, Korea reports the highest national frequency of physician visits worldwide. According to a 2017 OECD Health Statistics analysis, Korea reported the highest number of outpatient visits to physicians per capita among all OECD countries, with a rate of 16.0 per year in 2015 vs. the OECD average of 7.0 per year [24]. Taken together, the frequency of clinical visits made by Korean patients and 
the finding that patients with specific diseases face a higher risk of suicidal ideation support the suggestion that Korean physicians should screen for suicidal ideation in high-risk patients.

This study had some limitations. First, the cross-sectional design did not allow us to confirm the events that occurred between suicidal ideation and the visit to the primary care providers within the 2-week period prior to the survey. Second, the assessment of suicidal ideation was based only on the PHQ-9. Therefore, we may have underestimated the frequency of suicidal ideation and could not evaluate suicidal plans or attempts. Third, the ICD-10 codes were based on the history of self-reported visits to primary care providers. Therefore, further study is needed to evaluate these associations in greater detail. Despite these limitations, our study was the first to examine the association of suicidal ideation with musculoskeletal diseases and mental disorders in a nationally representative sample of primary care patients in the country with the highest suicide rate worldwide. Therefore, we believe that our analysis provides useful information.

\section{Abbreviations}

OECD: Organization for Economic Cooperation and Development

KNHANES: The Korean National Health and Nutrition Examination Survey

PHQ-9: Patient Health Questionnaire

ICD-10: Tenth International Classification of Diseases

BMl: Body mass index

\section{Declarations}

\section{Ethics approval and consent to participate}

This study was conducted according to the guidelines set forth in the Declaration of Helsinki. All procedures involving human subjects were approved by the Institutional Review Board of the Korea Centers for Disease Control and Prevention (2013-12EXP-03-5C). Written informed consent was obtained from all subjects.

\section{Consent for publication}

Not applicable

\section{Acknowledgements}


Availability of data and material

Data supporting the findings of this study were obtained from the Korea Centers for Disease Control and Prevention under license and are not publicly available. However, data are available from the authors upon reasonable request and with permission from the Korea Centers for Disease Control and Prevention.

\section{Competing interests}

All authors declare no conflicts of interest.

\section{Funding}

There are no sources of funding to declare.

\section{Authors' contributions}

$\mathrm{JH}$ Kim had full access to all the data in the study and takes responsibility for the integrity of the data and the accuracy of the data analysis. Study conception and design, Acquisition, analysis, or interpretation of data, drafting the manuscript, and critical revision of the manuscript for important intellectual content: JH Kim and G Lee.

\section{References}

1. Organization WH: Public health action for the prevention of suicide: a framework. 2012.

2. The suicide prevalences of OECD countries, URL: http://spckorea-stat.or.kr/noticelist.do (access: Sep 20, 2018) [http://spckorea-stat.or.kr/noticelist.do]

3. Public Health Service: The surgeon general's call to action to prevent suicide: Department of Health and Human Services, US Public Health Service; 1999.

4. Health Do, USA HS: National strategy for suicide prevention: Goals and objectives for action: na; 2001.

5. Bunney W, Kleinman A, Pellmar T, Goldsmith S: Reducing suicide: A national imperative: National Academies Press; 2002.

6. Luoma JB, Martin CE, Pearson JL: Contact with mental health and primary care providers before suicide: a review of the evidence. American Journal of Psychiatry 2002, 159(6):909-916.

7. Jordan P, Shedden-Mora MC, Löwe B: Predicting suicidal ideation in primary care: An approach to identify easily assessable key variables. General hospital psychiatry 2018, 51:106-111. 
8. Suicide Risk in Adolescents, Adults and Older Adults: Screening, URL: https://www.uspreventiveservicestaskforce.org/Page/Document/UpdateSummaryFinal/suicide-riskin-adolescents-adults-and-older-adults-screening (accessed: Sep 20, 2018)

9. Kweon S, Kim Y, Jang M-j, Kim Y, Kim K, Choi S, Chun C, Khang Y-H, Oh K: Data resource profile: the Korea national health and nutrition examination survey (KNHANES). International journal of epidemiology 2014, 43(1):69-77.

10. Kroenke K, Spitzer RL, Williams JB: The PHQ-9: validity of a brief depression severity measure. Journal of general internal medicine 2001, 16(9):606-613.

11. Han C, Jo SA, Kwak J-H, Pae C-U, Steffens D, Jo I, Park MH: Validation of the Patient Health Questionnaire-9 Korean version in the elderly population: the Ansan Geriatric study. Comprehensive psychiatry 2008, 49(2):218-223.

12. World Health Organization: The Asia-Pacific perspective: redefining obesity and its treatment. In.: Sydney: Health Communications Australia; 2000.

13. Hagströmer M, Oja P, Sjöström M: The International Physical Activity Questionnaire (IPAQ): a study of concurrent and construct validity. Public health nutrition 2006, 9(6):755-762.

14. Schulberg HC, Bruce ML, Lee PW, Williams Jr JW, Dietrich AJ: Preventing suicide in primary care patients: the primary care physician's role. General hospital psychiatry 2004, 26(5):337-345.

15. Wiborg JF, Gieseler D, Löwe B: Suicidal ideation in German primary care. General hospital psychiatry 2013, 35(4):366-369.

16. Brown GK, Beck AT, Steer RA, Grisham JR: Risk factors for suicide in psychiatric outpatients: a 20year prospective study. Journal of consulting and clinical psychology 2000, 68(3):371.

17. Nock MK, Borges G, Bromet EJ, Alonso J, Angermeyer M, Beautrais A, Bruffaerts R, Chiu WT, De Girolamo G, Gluzman S: Cross-national prevalence and risk factors for suicidal ideation, plans and attempts. The British Journal of Psychiatry 2008, 192(2):98-105.

18. Schulberg HC, Lee PW, Bruce ML, Raue PJ, Lefever JJ, Williams JW, Dietrich AJ, Nutting PA: Suicidal ideation and risk levels among primary care patients with uncomplicated depression. The Annals of Family Medicine 2005, 3(6):523-528.

19. Bomyea J, Lang AJ, Craske MG, Chavira D, Sherbourne CD, Rose RD, Golinelli D, Campbell-Sills L, Welch SS, Sullivan G: Suicidal ideation and risk factors in primary care patients with anxiety disorders. Psychiatry research 2013, 209(1):60-65.

20. Cavanagh JT, Carson AJ, Sharpe M, Lawrie SM: Psychological autopsy studies of suicide: a systematic review. Psychological medicine 2003, 33(3):395-405.

21. Krebber A, Buffart L, Kleijn G, Riepma I, De Bree R, Leemans C, Becker A, Brug J, van Straten A, Cuijpers P: Prevalence of depression in cancer patients: a meta-analysis of diagnostic interviews and self-report instruments. Psycho-Oncology 2014, 23(2):121-130.

22. Linton SJ: A review of psychological risk factors in back and neck pain. Spine 2000, 25(9):11481156. 
23. Choi SB, Lee W, Yoon J-H, Won J-U, Kim DW: Ten-year prediction of suicide death using Cox regression and machine learning in a nationwide retrospective cohort study in South Korea. Journal of affective disorders 2018, 231:8-14.

24. Ministry of Health and Welfare and Korea Institute for Health and Social Affairs: OECD Health Statics 2017 (abridgement). In.; 2017.

\section{Tables}

Table 1. Participants' general characteristics $(N=1,211)$

\begin{tabular}{|c|c|c|c|}
\hline & \multicolumn{2}{|c|}{ Suicidal ideation } & \multirow[b]{2}{*}{ P-value } \\
\hline & No $(n=1,057)$ & Yes $(n=154)$ & \\
\hline & $88.2(1.0)$ & $11.8(1.0)$ & \\
\hline Age (years, mean \pm SE) & $51.1 \pm 0.7$ & $55.1 \pm 2.3$ & .08 \\
\hline Sex (men), \% (SE) & $42.4(1.6)$ & $24.1(4.6)$ & $<.01$ \\
\hline Living (urban), \% (SE) & $82.9(2.8)$ & $77.0(4.5)$ & .11 \\
\hline Household income, \% (SE) & & & $<.01$ \\
\hline Low & $17.0(1.6)$ & $46.5(5.2)$ & \\
\hline Lower-middle & $25.1(1.8)$ & $27.0(4.6)$ & \\
\hline Upper-middle & $30.7(2.0)$ & $9.4(2.7)$ & \\
\hline High & $27.1(2.3)$ & $17.1(4.5)$ & \\
\hline Education (college degree or higher), \% (SE) & $64.0(1.7)$ & $37.9(5.4)$ & $<.01$ \\
\hline Occupation, \% (SE) & & & $<.01$ \\
\hline Manager, professional, office worker & $22.2(1.7)$ & $5.9(2.6)$ & \\
\hline Service, sales & $13.8(1.5)$ & $15.0(4.0)$ & \\
\hline Manual worker & $22.4(1.7)$ & $15.9(3.7)$ & \\
\hline Unemployed & $41.6(1.9)$ & $63.2(5.2)$ & \\
\hline Living together, \% (SE) & $73.9(1.6)$ & $50.4(5.2)$ & $<.01$ \\
\hline $\mathrm{BMI}, \%(\mathrm{SE})$ & & & .06 \\
\hline Under 18.5 kg/m² & $3.8(0.7)$ & $8.2(2.5)$ & \\
\hline Over $25 \mathrm{~kg} / \mathrm{m}^{2}$ & $33.1(1.9)$ & $34.7(4.3)$ & \\
\hline Current smoker, \% (SE) & $20.1(1.6)$ & $17.6(4.2)$ & .59 \\
\hline Heavy drinker, \% (SE) & $32.2(1.7)$ & $24.3(4.4)$ & .08 \\
\hline Adequate physical activity, \% (SE) & $14.7(1.3)$ & $8.3(2.9)$ & .10 \\
\hline Strength exercise, \% (SE) & $20.7(1.5)$ & $11.3(3.0)$ & .02 \\
\hline
\end{tabular}

$\mathrm{SE}$, standard error; BMI, body mass index.

Table 2. Diseases diagnosed by primary care providers and the self-reported underlying disease among participants per suicidal ideation $(\mathrm{N}=1,211)$ 


\begin{tabular}{lccc}
\hline & \multicolumn{2}{c}{ Suicidal ideation } & \\
\cline { 2 - 3 } & No $(\mathrm{n}=1,057)$ & Yes $(\mathrm{n}=154)$ & P-value \\
\hline Diseases diagnosed by primary care providers & & & \\
A00-B99 & $2.9(0.7)$ & $0.7(0.5)$ & .04 \\
\hline C00-C97 & $0.5(0.3)$ & $1.5(1.4)$ & .34 \\
\hline E00-E90 & $4.8(0.7)$ & $4.2(2.5)$ & .81 \\
\hline F00-F99 & $1.2(0.4)$ & $10.6(3.7)$ & $<.01$ \\
\hline G00-G99 & $1.3(0.4)$ & $2.5(1.5)$ & .30 \\
\hline H00-H59 & $5.0(0.8)$ & $5.2(1.9)$ & .90 \\
\hline H60-H95 & $2.1(0.7)$ & $1.7(1.1)$ & .80 \\
\hline I00-199 & $13.1(1.3)$ & $12.9(3.4)$ & .96 \\
\hline J00-J99 & $28.4(1.8)$ & $25.4(4.5)$ & .52 \\
\hline K00-K93 & $9.1(1.0)$ & $8.8(2.8)$ & .95 \\
\hline L00-L99 & $6.0(0.9)$ & $8.2(3.0)$ & .44 \\
\hline M00-M99 & $21.1(1.6)$ & $30.3(4.2)$ & .03 \\
\hline N00-N99 & $3.1(0.6)$ & $3.2(2.0)$ & .96 \\
\hline O00-099 & $0.1(0.1)$ & $\mathrm{N} / \mathrm{A}$ & \\
\hline R00-R99 & $3.0(0.6)$ & $4.4(1.8)$ & .41 \\
\hline S00-T98 & $5.1(0.9)$ & $2.2(1.2)$ & .11 \\
\hline Z00-Z99 & $9.7(1.2)$ & $7.0(2.4)$ & .35 \\
\hline Self-reported underlying diseases & & & \\
Hypertension & $27.2(1.7)$ & $36.0(4.8)$ & .04 \\
\hline Diabetes mellitus & $10.3(0.9)$ & $11.5(2.8)$ & .68 \\
\hline Dyslipidemia & $18.6(1.4)$ & $24.8(4.0)$ & .11 \\
\hline Stroke & $2.4(0.6)$ & $6.7(2.2)$ & $<.01$ \\
\hline Cardiovascular disease & $2.2(0.4)$ & $4.5(1.7)$ & .09 \\
\hline Depression & $6.0(0.9)$ & $23.4(4.1)$ & $<.01$ \\
\hline Cancer & $5.3(0.8)$ & $4.5(1.7)$ & .68 \\
\hline
\end{tabular}

N/A, not applicable. A00-B99, certain infectious and parasitic diseases; C00-C97, neoplasms; E00-E90, endocrine, nutritional, and metabolic diseases; F00-F99, mental and behavioral disorders; G00-G99, diseases of the nervous system; $\mathrm{H0O}-\mathrm{H} 59$, diseases of the eye and adnexa; $\mathrm{H60}-\mathrm{H} 95$, diseases of the ear and mastoid process; 100-199, diseases of the circulatory system; J00-J99, diseases of the respiratory system; K00-K93, diseases of the digestive system; L00-L99, diseases of the skin and subcutaneous tissue; M00-M99, diseases of the musculoskeletal system and connective tissue; N00N99, diseases of the genitourinary system; 000-099, pregnancy, childbirth, and the puerperium; R00R99, symptoms, signs and abnormal clinical and laboratory findings, not elsewhere classified; S00-T98, injury, poisoning, and certain other consequences of external causes; Z00-Z99, Factors influencing health status and contact with health services.

Table 3. The association between the disease diagnosed by primary care providers and suicidal ideation 


\begin{tabular}{lcl}
\hline & Odds ratio (95\% confidence interval) & P-value \\
\hline ICD-10 code & & \\
C00-C97 & $8.323(1.010-68.562)$ & $<.05$ \\
\hline F00-F99 & $6.194(1.718-22.326)$ & $<.01$ \\
\hline M00-M99 & $1.931(1.089-3.425)$ & .02 \\
\hline S00-T98 & $0.171(0.038-0.773)$ & .02 \\
\hline
\end{tabular}

ICD, International Classification of Diseases. C00-C97, neoplasms; F00-F99, mental and behavioral disorders; M00-M99, diseases of the musculoskeletal system and connective tissue; S00-T98, injury, poisoning, and certain other consequences of external causes. 